\title{
COMPORTAMIENTO DE LAS VARIABLES DASOMÉTRICAS EN PLANTACIONES DE Pinus contorta Doug. Ex Loud., BAJO MANEJO SILVOPASTORAL Y FORESTAL EN LA REGIÓN DE AYSÉN, CHILE
}

\begin{abstract}
Sotomayor Alvaro ${ }^{1}$, Moya Ivan ${ }^{1}$, Acuña Bernardo ${ }^{1}$
RESUMEN

Los suelos agrícolas productivos de la región de Aysén, constituidos por 746.807 ha, son destinados preferentemente a la producción ganadera, con un $88,7 \%$ de éstos bajo este uso. Sólo 43.271 ha son destinadas a plantaciones forestales, principalmente con Pinus ponderosa, Pinus contorta y Pseudotsuga menziesii.

El presente estudio entrega resultados del comportamiento de la especie Pinus contorta,

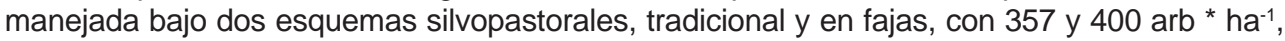
respectivamente, en relación a un sistema de plantación con manejo forestal con 800 arb ha-1, todos podados al $40 \%$ de la altura total,

Los resultados obtenidos arrojan que los árboles ordenados bajo ordenamiento silvopastoral tuvieron un mejor desarrollo en diámetro a la altura del pecho (DAP), una menor área basal $(A B)$, y una menor cobertura de copa por unidad de superficie, en relación al sistema forestal. A su vez, hubo diferencias significativas en altura media total entre el tratamiento silvopastoral tradicional y el forestal, y no existieron diferencias en relación al cociente de forma y al diámetro de ramas.
\end{abstract}

No se presentaron daños por efecto del viento, ni por efecto del pastoreo, en los tratamientos silvopastorales.

Palabras clave: Sistemas silvopastorales, Patagonia, Pinus contorta

\section{SUMMARY}

Productive agricultural soils of the Aysen Region, consisting of 746,807 ha, are dedicated primarily to livestock production, with $88.7 \%$ of them for this use in relation to agricultural land use. Only 43,271 ha are forest plantations, mainly Pinus ponderosa, Pinus contorta and Pseudotsuga menziessi.

This study provides results of the behavior of the species Pinus contorta, managed under two silvopastoral schemes, traditional and in strips, with 357 and 400 trees $^{-1}{ }^{-1}$, respectively, in relation to forest management system with 800 trees $^{*}$ ha $^{-1}$, all pruned to $40 \%$ of the total height.

The results shows that trees ordered under silvopastoral system had a better development

1 Instituto Forestal (INFOR) asotomay@infor.cl 
in DAP, lower basal area (AB) and canopy cover per hectare, compared to the forest system. In turn, significant differences in average height between traditional silvopastoral treatment and forest, and there were no differences regarding the local form quotient and diameter of branches.

There were no effects of wind and by grazing in the evaluated silvopastoral treatments.

Key words: Silvpastoral systems, Patagonia, Pinus contorta

\section{INTRODUCCIÓN}

El principal uso de los suelos agrícolas de la región de Aysén son las praderas naturales, destinadas al uso ganadero extensivo, con un $88,7 \%$ de la superficie agrícola utilizada, de las cuales el 56,5\% pertenece a grandes propietarios (INE, 1997). El otro gran uso es el forestal, donde la región de Aysén posee la mayor superficie nacional de bosque nativo en Chile, con 4,8 millones de hectáreas; esto se contrasta con las menores superficies de plantaciones del país, con solo 43 mil hectáreas (INFOR, 2008).

Las bajas tasas de forestación, 3 mil ha anuales en los últimos 5 años, se deben a la poca aceptación de los ganaderos por actividades de forestación tradicional, ya que usualmente éstos la perciben como una competencia con sus usos productivos agrícolas tradicionales. Esta visión de los productores ganaderos, es similar a lo que ocurre en otras regiones del mundo (Arnold, 1983). El cambio de percepción y actitud de los productores ganaderos de ésta región, frente a la reintroducción de árboles en su sistema productivo en una forma complementaria a la ganadería, es un proceso lento, dado que el manejo de los recursos agrícolas y de suelos está firmemente arraigado en el sistema cultural y productivo de los agricultores (Arnold, 1983; Longhurst, 1983; Sotomayor, 1989)

El uso de sistemas silvopastorales, es una práctica que se ha estado estudiando en los últimos años por el Instituto Forestal (INFOR) y el Instituto de Investigaciones Agropecuarias (INIA), para reintroducir el árbol en los campos en una forma amigable con los sistemas productivos ganaderos y con la cultura productiva de los agricultores y ganaderos de Aysén. De esta forma, se espera entregar una nueva forma de manejo productivo de los suelos, para mejorar la productividad de éstos, reducir su nivel de erosión, y protegerlos del factor erosivo más importante en esa región, que es el viento (Teuber y Ganderatz, 2009).

Los sistemas silvopastorales son una forma de aplicación de los sistemas agroforestales, y se definen como aquella práctica agroforestal que combina árboles, con praderas y producción de ganado en un mismo sitio, en forma deliberada, con el objetivo de producir alimento para los animales, productos derivados del ganado, y productos forestales como madera y pulpa, y otros como leña, carbón, miel, frutos y hongos (Sotomayor, 1990a).

En este tipo de sistemas integrados de producción, los árboles pueden producir madera aserrable, postes, leña, forraje para el ganado y frutas, a la vez que ayudan a mejorar la producción de pasto y ganado, reduciendo la influencia de los vientos y otorgando sombra (Sotomayor, 1990a; Murgeitio, 2009). Los árboles pueden proveer madera, como elemento económico y ayudar a controlar los procesos de erosión (Sotomayor 2009). A su vez, el ganado

266 / Ciencia e Investigación Forestal - Instituto Forestal / Chile 
provee un ingreso anual, controla el desarrollo de las malezas y reduce la competencia entre forraje y árboles (Sotomayor, 2009; Murgeitio, 2009).

En un sentido económico, la integración que logra el manejo silvopastoral, permite la producción de madera de alta calidad, cuando es manejado correctamente, generando ingresos de mediano y largo plazo, a la vez que provee un ingreso de corto plazo, proveniente de las operaciones ganaderas y de raleos intermedios. Estos ingresos permiten, a su vez, financiar los costos asociados al manejo forestal, como son principalmente raleo y poda, y el manejo de los desechos (Sotomayor, 1989; Sotomayor, 1990a).

Sin embargo, el manejo del sistema silvopastoral es más complejo que un sistema productivo monoespecífico, pues en él se produce la interacción entre los componentes herbáceos, arbóreos (y/o arbustivos) y animal (Garret y Buck, 1997; Garret et al., 2004; Sotomayor, 2009; Mead, 2009). En este sentido, los manejos asociados deben estar orientados a minimizar la competencia entre las estratas arbóreas y herbáceas, así como el daño potencial que puede provocar la presencia de animales en las plantaciones forestales (Lewis, 1973).

En términos generales, el mayor conflicto entre las estratas arbórea y herbácea dice relación con la competencia por agua y nutrientes, especialmente en el establecimiento de las plantaciones forestales, y la competencia por luz directa (Anderson et al., 1988), la que aumenta proporcionalmente con el desarrollo de los árboles y el cierre del dosel (Mead, 2009). Por otro lado, el efecto de los animales se relaciona directamente con el ramoneo y pisoteo de las plantas, situación que es especialmente sensible durante los primeros años de vida de la planta (Monfore, 1983; Sotomayor, 1990c).

Si bien, se han reportado diversos beneficios de la integración de estos componentes productivos, también existen estudios que informan una menor producción total de la cubierta arbórea en densidades silvopastorales (Sotomayor 1990a; Sotomayor y Cabrera 2008), pero árboles de mayor tamaño y valor (Sotomayor y Cabrera, 2008). Otro aspecto a considerar en sistemas silvopastorales es el manejo animal, para evitar daños por ramoneo y pisoteo al establecimiento de las plantas y durante su rotación, dado que puede afectar el éxito del proyecto (Tustin et al., 1979; Doesher et al., 1987; Krueger, 1987).

\section{OBJETIVO}

El objetivo es analizar el comportamiento de las variables dasométricas en plantaciones de Pinus contorta, bajo manejo forestal y silvopastoral.

\section{MATERIAL Y METODO}

Los tratamientos instalados para caracterizar los parámetros forestales se describen en el Cuadro N 1. La plantación original de Pinus contorta fue establecida el año 1991, y no había sido manejada hasta el momento del establecimiento de los ensayos en evaluación. 


\section{Cuadro $\mathrm{N}^{\circ} 1$ \\ TRATAMIENTOS EVALUADOS, FORESTAL MANEJADO Y SILVOPASTORALES}

\begin{tabular}{|c|c|c|c|c|c|c|c|}
\hline \multirow{2}{*}{ № } & \multirow{2}{*}{ Nombre y descripción tratamiento } & \multicolumn{3}{|c|}{$\begin{array}{l}\text { Densidad } \\
\text { (arb ha-1) }\end{array}$} & \multicolumn{2}{|c|}{$\begin{array}{c}\text { Altura de poda } \\
2004(\mathrm{~m})\end{array}$} & \multirow{2}{*}{$\begin{array}{l}\text { DSM } \\
\text { (cm) } \\
\star \star \star \star \star\end{array}$} \\
\hline & & Inicial & $2004^{*}$ & Final $^{* \star}$ & $2004^{\star}$ & $\underset{* * \star}{\text { Final }}$ & \\
\hline $\mathrm{T} 1$ & $\begin{array}{l}\text { Sistema Forestal Manejado, sin mejoramiento de la } \\
\text { pradera mediante fertilización. }\end{array}$ & 1.514 & 816 & 400 & 1,97 & 6,0 & 20,0 \\
\hline T2 & $\begin{array}{l}\text { Sistema Silvopastoral Tradicional, con pradera natural } \\
\text { mejorada con fertilización. }\end{array}$ & 1.514 & 357 & $\begin{array}{l}200- \\
250\end{array}$ & 2,01 & 6,0 & 20,0 \\
\hline T3 & $\begin{array}{l}\text { Sistema Silvopastoral en Fajas, con pradera natural } \\
\text { mejorada con fertilización. }\end{array}$ & 1.514 & 400 & $\begin{array}{l}200- \\
250\end{array}$ & 2,09 & 6,0 & 20,0 \\
\hline
\end{tabular}

DSM: diámetro objetivo sobre verticilo podado, en la oportunidad correspondiente.

*: Densidad y poda real lograda después de intervención año 2004

**: Densidad final proyectada a la rotación de 35-40 años.

***: Poda final proyectada a la rotación de 35-40 años.

**** Diámetro sobre muñón podado proyectado

Para el análisis del comportamiento de las variables dasométricas con respecto a la densidad y manejo, se midieron y calcularon los parámetros forestales en marzo del año 2004, para obtener la línea base de comparación, y luego medidos cada año, entre marzo-abril de los años 2005 a 2008.

\section{Parámetros Forestales Evaluados}

- $\quad$ DAP (diámetro altura del pecho): Diámetro del fuste a una altura de 1,3 m, medido en todos los árboles de la parcela.

- $\quad$ DSM (diámetro sobre muñón podado): Consiste en el diámetro medido sobre el último verticilo superior de la sección podada, medido en todos los árboles de la parcela.

- $\quad$ AB (área basal media): Área basal media, en base a todos los árboles de la parcela.

- $\quad$ H media total (altura media total del árbol): Altura media total de todos los árboles de la parcela, la cual fue medida con una vara graduada.

- $\quad$ H poda (altura de poda): Altura de poda al primer verticilo no podado, medida con una vara graduada, en todos los árboles de la parcela.

- $\quad$ Cociente de forma local (CFL): El CFL se evaluó en la temporada 2008-2009. Para ello se diseño un cociente de forma local, dado que otros factores de forma consideran mediciones en altura a los 5,27 m (Cociente de Forma de Girard), lo cual no fue conveniente por la baja altura al momento del inicio del estudio. Para ello se midió el diámetro a 3,20 m de la altura del árbol (Nota: largo de trozas usadas para madera de especies de coníferas en aserraderos locales) y el DAP, sobre los 100 árboles de mayor altura por hectárea de cada tratamiento, equivalente parcela, y se calculó el cociente de forma local (CFL) utilizando 
la siguiente fórmula:

$$
\mathrm{CFL}=\mathrm{D}_{3,2 \mathrm{~m}} \text { (diámetro a 3,20 m árbol)/DAP(diámetro a 1,3 m) }
$$

(Formula 1. Cociente de forma local)

- Diámetro Ramas: Se midió el diámetro de ramas del primer verticilo no podado. En este se midió el diámetro de las tres ramas de mayor diámetro del verticilo, en 8 árboles al azar de cada parcela. Se calculó el promedio de estas ramas podadas, y se compara entre tratamientos.

- Cobertura de copa: La cobertura de la copa fue calculada en base al siguiente procedimiento (Figura $\mathrm{N}^{\circ} 1$ ):

- Diámetro de copa orientación norte-sur (D1): Se midió el diámetro total de la copa entre los extremos de las ramas verdes norte-sur, proyectado sobre la superficie del suelo, en todos los arboles de la parcela.

- Diámetro de copa orientación este-oeste (D2): Se midió el diámetro total de la copa entre los extremos de las ramas verdes este-oeste, proyectado sobre la superficie del suelo, en todos los arboles.

- Calculo: Con estas medidas se calculó el diámetro medio, y con este se calcula el área de una circunferencia, y la superficie en porcentaje que queda bajo la proyección de las copas de los árboles, de la siguiente forma, y que da origen a la formula 2 de cobertura de copa.

○ Diámetro medio de ancho de copa: $d=(D 1+D 2)$

○ Área de Cobertura bajo copa: $A c o p a=\pi(d / 2)^{2}$

$$
\text { Ccopa }(\%)=\left(\mathrm{N} / \mathrm{ha}{ }^{*} \text { Acopa }\right) / 10000 * 100
$$

(Formula 2. Calculo cobertura de copa) 


\section{Figura $\mathrm{N}^{\circ} 1$ \\ DESCRIPCIÓN MEDICIÓN COBERTURA DE COPA (Ccopa\%).}
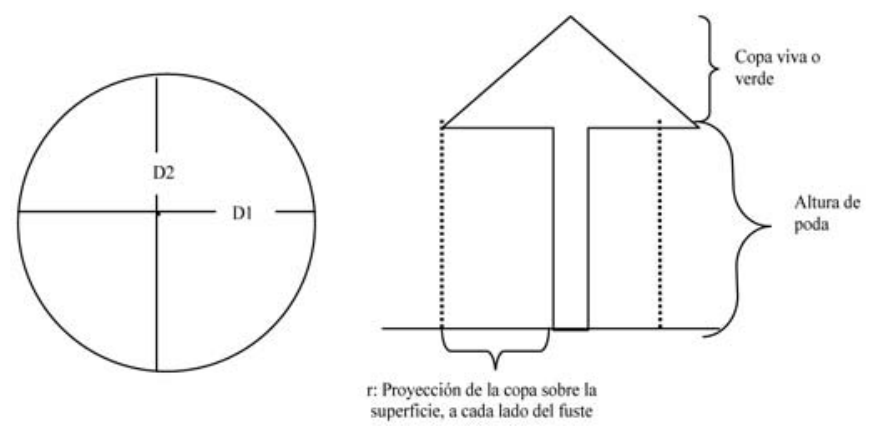

- $\quad$ Otras variables: Además, se evaluaron los siguientes aspectos que son de interés para el manejo del sistema silvopastoral:

- Daños por pastoreo: en la parcelas de medición se midió el daño de los animales sobre los árboles, con los siguientes criterios:

Daño en la corteza por fricción o por efecto de cuernos: № de árboles dañados.

Daño por inclinación de los árboles por fricción o tránsito: № de árboles dañados.

Ramoneo en las ramas bajas: № de árboles ramoneados.

Otros daños encontrados.

Evaluación (\% de árboles dañados): Se evalúa el daño total por efecto de los animales.

Daño por viento: se contabilizara los árboles inclinados o volteados por efecto viento.

Cobertura de desechos (\%): se contabilizó la superficie cubierta de desechos provenientes de la poda y raleo sobre la pradera.

\section{Diseño Experimental}

Para la evaluación de los parámetros forestales en los tratamientos con presencia de árboles de la especie Pinus contorta, se utilizó un diseño de parcelas permanentes distribuidas al azar, con medidas repetidas en el tiempo, de la siguiente forma: 
T1: Forestal Manejado: Se utilizaron 3 parcelas rectangulares de $200 \mathrm{~m}^{2}$, distribuidas al azar dentro del área del tratamiento, que es de $5.000 \mathrm{~m}^{2}$.

T2: Silvopastoral Tradicional: Se utilizaron 3 parcelas rectangulares de $1.008 \mathrm{~m}^{2}$ (24x42 m), distribuidas al azar dentro de las 5,3 ha del tratamiento, con orientación este-oeste.

T3: Silvopastoral en Fajas. Tres parcelas rectangulares, de $1.008 \mathrm{~m}^{2}$ (24 x $42 \mathrm{~m}$ ), distribuidas al azar dentro de las 5,5 ha del tratamiento. Las parcelas en este caso están orientadas en el sentido de la pendiente, este-oeste, de forma que puedan incluir en estas dos fajas de árboles, y un área completa de pradera entre estas fajas.

\section{Evaluación Estadística de los Resultados}

Para realizar los análisis estadísticos, se decidió trabajar con Modelos Estadísticos Lineales Mixtos, para atender la falta de alguno(s) de los supuestos clásicos que requiere un análisis de varianza tradicional, y que son:

Los datos deben presentar una distribución normal

Independencia de los datos

Heterogeneidad de varianza.

Para el análisis de las variables forestales se realizó un análisis de mediciones repetidas en el tiempo, o también conocido como análisis longitudinal. Estas mediciones repetidas son dependientes, lo que origina el no cumplimiento de la independencia de datos (las mediciones del año 2 dependen de las mediciones del año 1 y así sucesivamente), dado lo cual se hace necesario utilizar modelos mixtos para su evaluación. Esta metodología de análisis se utiliza para todos los parámetros forestales.

Por ello se consideró, para cada individuo y parámetro, mediciones consecutivas en el tiempo, dependiendo de los años de evaluaciones.

Este análisis permite identificar tanto el efecto medio del tratamiento como el efecto del tiempo y la interacción tiempo por tratamiento, que corresponden a las tres hipótesis evaluadas, y que por lo tanto generan tres valores $p$.

El modelo de análisis estadístico utilizado para analizar las variables forestales, pratenses y animales, corresponde a modelo expuesto en fórmula 3.

$$
Y=\mu+T+t+\left(T^{*} t\right)+P+E
$$

(Formula 3. Modelo de análisis estadístico) 
Donde:

$$
\begin{aligned}
& \mathrm{Y}=\text { Modelo } \\
& \mu=\text { Constante } \\
& \mathrm{T}=\text { Tratamiento } \\
& \mathrm{t}=\text { Tiempo } \\
& \mathrm{P}=\text { Parcela } \\
& \mathrm{E}=\text { Error }
\end{aligned}
$$

\section{RESULTADOS}

\section{Diámetro Altura del Pecho (DAP)}

En el Cuadro $\mathrm{N}^{\circ} 2$ y en la Figura $\mathrm{N}^{\circ} 2$, se muestra la evolución del DAP entre los años 2004 y 2008.

\section{Cuadro $\mathrm{N}^{\circ} 2$ \\ DESARROLLO DEL DAP Pinus contorta AÑOS 2004 A 2008}

\begin{tabular}{|ll|c|c|c|c|c|c|}
\hline \multirow{3}{*}{\multicolumn{2}{|c|}{ Tratamiento }} & \multicolumn{5}{c|}{ DAP (cm) } & \multirow{2}{*}{$\begin{array}{c}\text { Incremento } \\
\mathbf{2 0 0 4 - 2 0 0 8}(\%)\end{array}$} \\
\cline { 3 - 8 } & $\mathbf{2 0 0 4}$ & $\mathbf{2 0 0 5}$ & $\mathbf{2 0 0 6}$ & $\mathbf{2 0 0 7}$ & $\mathbf{2 0 0 8}$ & 33,1 \\
\hline T1 & Forestal Manejado & 12,7 & 13,6 & 14,8 & 16,0 & 16,9 & 48,1 \\
\hline T2 & Silvopastoral Tradicional & 12,9 & 14,2 & 16,0 & 17,8 & 19,1 & 38,5 \\
\hline T3 & Silvopastoral en Fajas & 13,0 & 14,1 & 15,6 & 17,1 & 18,0 & 3 \\
\hline
\end{tabular}

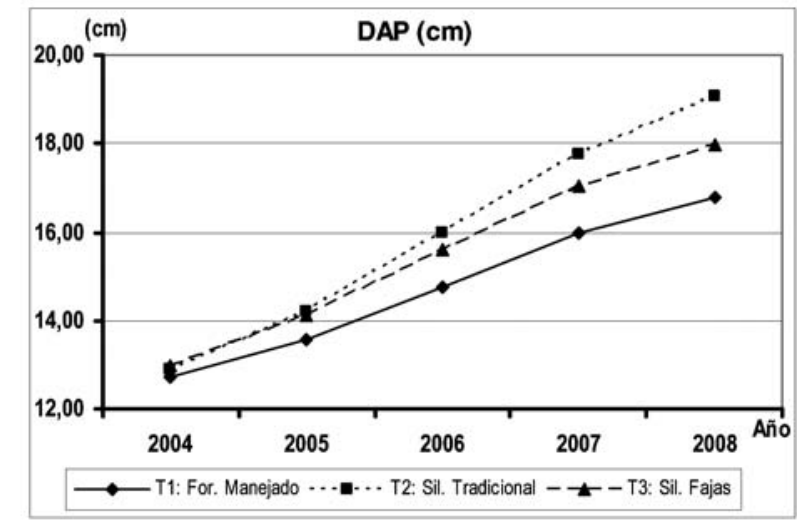

Figura $\mathrm{N}^{\circ} 2$

EVOLUCIÓN DEL DAP Pinus contorta AÑOS 2004 A 2008

Al comenzar el estudio el año 2004, los tres tratamientos tenían un DAP similar y cercano a los $13 \mathrm{~cm}$, lo cual fue variando en el transcurso de los años. Es así que al año 2008, 
el tratamiento de mayor diámetro es el T2, silvopastoral tradicional, superando en $1,1 \mathrm{~cm}$ al tratamiento en fajas y en 2,28 cm al tratamiento forestal manejado.

Lo anterior se debe fundamentalmente al efecto de la densidad ( $\left.\operatorname{arb~ha}^{-1}\right)$ y a la distribución de los árboles en la superficie. Es así, que en el tratamiento T2 los árboles tienen un mejor desarrollo ya que han tenido un mayor espacio para su crecimiento, en relación a los otros dos tratamientos. Estos están distribuidos uniformemente en la superficie, con un distanciamiento promedio de 5,3 m entre ellos contra 3,5 m en forestal y 2,2 m en el área interior de la faja en dicho tratamiento silvopastoral.

Si bien el tratamiento silvopastoral en fajas tiene una densidad similar que el tradicional, 400 y 357 arb ha-1, respectivamente, en el primer tratamiento los árboles se concentran en una faja de terreno de menor área. Esta área de la faja tiene un ancho promedio de $6 \mathrm{~m}$ con un distanciamiento entre árboles en el sector interno de 2,2 m entre ellos y, entre los árboles exteriores entre las fajas, el distanciamiento es de 21,0 m. La situación anterior genera una mayor competencia entre los árboles, tanto aérea como radicular, y como resultado existe un menor desarrollo y una alta variabilidad entre los árboles exteriores e interiores. El menor DAP en el sistema forestal tiene su explicación en la mayor densidad de este (816 arb ha-1) respecto a los sistemas silvopastorales.

Para el análisis estadístico del DAP, se probaron cuatro modelos que ajustan varianzas heterogéneas:

Autoregresivo Heterogeneo (HA)

Autoregresivo de Orden 1 (AR1)

De Simetría Compuesta (CS)

De Simetría Compuesta Heterocedástica (CSH).

Los cuatro modelos alcanzaron los criterios de convergencia requeridos por SAS. El Test de Levene indicó que hay homogeneidad de varianzas para el DAP en todos los años evaluados.

Para seleccionar el mejor modelo para esta variable, se utilizó el criterio de información de Akaike (Indice AIC = -2log $(M V)+2 n(p+q+1) /(n-(p+q)-2)$, donde MV: máxima verosimilitud, q: número de parámetros de la estructura de covarianza, $\mathrm{p}$ : rango de la matriz de diseño x, y n: número de observaciones. En este criterio, a menor valor, mejor será el ajuste (Carrero et al. 2008). Este procedimiento fue aplicado a todas las variables forestales. De acuerdo al índice AIC, el mejor modelo corresponde al Modelo CSH, por presentar el menor valor AIC, dado lo cual fue el utilizado para este estudio.

Se considera que para medidas repetidas en el tiempo y trabajos de campo, un $\infty_{<=0,05}$ representa diferencias significativas. En el Cuadro $\mathrm{N}^{\circ} 3$ se observa que el efecto tiempo y tratamiento*tiempo son significativos, y tratamiento y parcela no son significativos para la variable DAP. De acuerdo a estos resultados, se analiza el efecto tratamiento*tiempo. 


\section{Cuadro $\mathrm{N}^{\circ} 3$ \\ RESULTADOS DEL MODELO CSH PARA DAP}

\begin{tabular}{|l|c|c|c|c|}
\hline Efecto & GL & DF & Valor $\mathbf{F}$ & Pr $>$ F \\
\hline Tratamiento & 2 & 4 & 2,04 & 0,2447 \\
\hline Tiempo & 4 & 1368 & 252,79 & $<, 0001$ \\
\hline Parcela & 2 & 4 & 0,11 & 0,8997 \\
\hline Tratamiento*Tiempo & 12 & 1368 & 101,88 & 0,0017 \\
\hline
\end{tabular}

De acuerdo a los resultados expuestos en Cuadro $N^{\circ} 4$ y Figura $N^{\circ} 2$, se presentan diferencias significativas entre los tratamientos en el tiempo para DAP.

En la Figura $\mathrm{N}^{\circ} 2$ se observa que en el transcurso de los años, las curvas de crecimiento del DAP entre tratamientos se separan a partir del segundo año de medición. Entre el tratamiento silvopastoral tradicional y el forestal, se presentan diferencias significativas a partir del año 2007 hasta el año 2008 (Cuadro $N^{\circ} 4$ ), y entre el tratamiento silvopastoral en fajas y el forestal, no se presentaron diferencias, aunque el último año ya se comienzan a observar diferencias, aunque no significativas.

Durante todo el tiempo de evaluación, no se presentaron diferencias entre los dos tratamientos silvopastorales, aunque se observa también la tendencia, a medida que aumenta la edad, de obtener mayores diferencias en DAP en los tratamientos silvopastorales con respecto a tratamiento forestal, producto de la menor densidad.

\section{Cuadro $\mathrm{N}^{\circ} 4$}

\section{COMPARACIONES PARA TRATAMIENTO*TIEMPO PARA DAP}

\begin{tabular}{|l|l|l|l|}
\hline Tratamiento & Tratamiento & Año & Pr $>|\mathbf{t}|$ \\
\hline T2: Sil. Tradicional & T3: Sil. Fajas & 2004 & 0,9011 \\
\hline T2: Sil. Tradicional & T1: M. Forestal & 2004 & 0,5923 \\
\hline T3: Sil. Fajas & T1: M. Forestal & 2004 & 0,5139 \\
\hline T2: Sil. Tradicional & T3: Sil. Fajas & 2005 & 0,9841 \\
\hline T2: Sil. Tradicional & T1: M. Forestal & 2005 & 0,3179 \\
\hline T3: Sil. Fajas & T1: M. Forestal & 2005 & 0,3234 \\
\hline T2: Sil. Tradicional & T3: Sil. Fajas & 2006 & 0,5981 \\
\hline T2: Sil. Tradicional & T1: M. Forestal & 2006 & 0,0776 \\
\hline T3: Sil. Fajas & T1: M. Forestal & 2006 & 0,2028 \\
\hline T2: Sil. Tradicional & T3: Sil. Fajas & 2007 & 0,3652 \\
\hline T2: Sil. Tradicional & T1: M. Forestal & 2007 & 0,0164 \\
\hline T3: Sil. Fajas & T1: M. Forestal & 2007 & 0,1313 \\
\hline T2: Sil. Tradicional & T3: Sil. Fajas & 2008 & 0,1298 \\
\hline T2: Sil. Tradicional & T1: M. Forestal & 2008 & 0,0011 \\
\hline T3: Sil. Fajas & T1: M. Forestal & 2008 & 0,0756 \\
\hline
\end{tabular}




\section{-Área Basal}

Para el caso del AB, como indicador de ocupación del sitio, se obtuvieron los siguientes resultados (Cuadro $\mathrm{N}^{\circ} 5$ ).

\section{Cuadro $\mathrm{N}^{\circ} 5$}

\section{DESARROLLO DEL AB ENTRE LOS AÑOS 2004 A 2008}

\begin{tabular}{|l|c|c|c|c|c|c|}
\hline \multirow{2}{*}{ Tratamiento } & \multicolumn{5}{|c|}{$\begin{array}{c}\text { AB } \\
\left(\mathbf{m}^{\mathbf{2}} \mathbf{h a}^{-1}\right)\end{array}$} & $\begin{array}{c}\text { Incremento } \\
\mathbf{2 0 0 4 - 2 0 0 8} \\
(\%)\end{array}$ \\
\cline { 2 - 6 } & $\mathbf{2 0 0 4}$ & $\mathbf{2 0 0 5}$ & $\mathbf{2 0 0 6}$ & $\mathbf{2 0 0 7}$ & $\mathbf{2 0 0 8}$ & 72,6 \\
\hline T1: For. Manejado & 10,6 & 12,0 & 14,2 & 16,6 & 18,3 & 114,6 \\
\hline T2: Sil. Tradicional & 4,8 & 5,8 & 7,3 & 9,1 & 10,3 & 103,7 \\
\hline T3: Sil. Fajas & 5,4 & 6,4 & 8,3 & 9,9 & 11,0 & \\
\hline
\end{tabular}

EI AB, influenciada fuertemente por la densidad y luego por el DAP, indica que el tratamiento $\mathrm{T} 1$ obtiene una mayor $\mathrm{AB}$ con $18,3 \mathrm{~m}^{2} \mathrm{ha}^{-1}$, producto de sus 816 árboles. Este valor (año 2008) es superior en un $70 \%$ a los sistemas silvopastorales, que obtienen un $A B$ entre 10,3 y $11 \mathrm{~m}^{2} \mathrm{ha}^{-1}$, influenciados por una densidad menor, entre 357 a 400 arb ha-1.

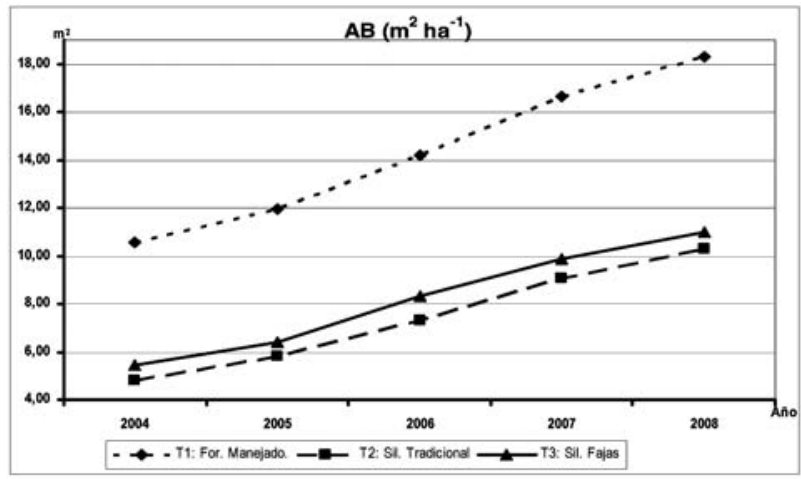

Figura $\mathrm{N}^{\circ} 3$

\section{EVOLUCIÓN DEL AB AÑOS 2004 A 2008}

Para analizar este parámetro, se probaron los mismos cuatro modelos que ajustan varianzas heterogéneas empleados para el análisis del DAP. Los cuatro modelos alcanzaron los criterios de convergencia requeridos por SAS.

El Test de Levene indicó que hay homogeneidad de varianzas para el AB en todos los años evaluados. Los resultados indican que el mejor modelo corresponde al Modelo UN, que presenta los menores valores AIC. 


\section{Cuadro $\mathrm{N}^{\circ} 6$}

\section{RESULTADOS DEL MODELO UN PARA AB}

\begin{tabular}{|l|c|c|c|c|}
\hline Efecto & GL & DF & Valor $\mathbf{F}$ & Pr $>$ F \\
\hline Tratamiento & 2 & 4 & 28,01 & 0,0044 \\
\hline Tiempo & 4 & 24 & 428,45 & $<, 0001$ \\
\hline Parcela & 2 & 4 & 0,70 & 0,5470 \\
\hline Tratamiento*Tiempo & 8 & 24 & 6,06 & 0,0003 \\
\hline
\end{tabular}

Los efectos tratamiento, tiempo, y tratamiento*tiempo (Cuadro $\mathrm{N}^{\circ} 6$ ) son significativos para la variable $A B$, y sólo el efecto parcela no es significativo.

En relación a la interacción tratamiento*tiempo, tal como se expone en el Cuadro $\mathrm{N}^{\circ}$ 7, durante todos los años de evaluación existen diferencias significativas entre el tratamiento forestal en relación a los tratamientos silvopastorales, producto del mayor número de árboles por unidad de superficie presente en ese tratamiento.

También se determinó que no existen diferencias significativas entre los tratamientos silvopastorales. No obstante, el tratamiento T3 presenta AB mayores que T2 a través del tiempo (Figura $\mathrm{N}^{\circ} 4$ ).

\section{Cuadro $N^{\circ} 7$}

\section{COMPARACIONES ENTRE TRATAMIENTO*TIEMPO PARA AB}

\begin{tabular}{|l|l|l|l|}
\hline Tratamiento & Tratamiento & Año & Pr $>|\mathbf{t}|$ \\
\hline T1: M. Forestal & T2: Sil. Tradicional & 2004 & $<0,001$ \\
\hline T1: M. Forestal & T3: Sil. Fajas & 2004 & $<0,001$ \\
\hline T2: Sil. Tradicional & T3: Sil. Fajas & 2004 & 0,5438 \\
\hline T1: M. Forestal & T2: Sil. Tradicional & 2005 & $<0,001$ \\
\hline T1: M. Forestal & T3: Sil. Fajas & 2005 & $<0,001$ \\
\hline T2: Sil. Tradicional & T3: Sil. Fajas & 2005 & 0,5775 \\
\hline T1: M. Forestal & T2: Sil. Tradicional & 2006 & $<0,001$ \\
\hline T1: M. Forestal & T3: Sil. Fajas & 2006 & $<0,001$ \\
\hline T2: Sil. Tradicional & T3: Sil. Fajas & 2006 & 0,3511 \\
\hline T1: M. Forestal & T2: Sil. Tradicional & 2007 & $<0,001$ \\
\hline T1: M. Forestal & T3: Sil. Fajas & 2007 & $<0,001$ \\
\hline T2: Sil. Tradicional & T3: Sil. Fajas & 2007 & 0,4475 \\
\hline T1: M. Forestal & T2: Sil. Tradicional & 2008 & $<0,001$ \\
\hline T1: M. Forestal & T3: Sil. Fajas & 2008 & $<0,001$ \\
\hline T2: Sil. Tradicional & T3: Sil. Fajas & 2008 & 0,4993 \\
\hline
\end{tabular}




\section{-Altura Total Media}

La altura total media se midió sobre el total de los árboles de cada parcela permanente, y sus resultados se presentan en el Cuadro $\mathrm{N}^{\circ} 8$.

\section{Cuadro $\mathrm{N}^{\circ} 8$}

\section{ALTURA MEDIA TOTAL ENTRE LOS AÑOS 2004 A 2008}

\begin{tabular}{|c|c|c|c|c|c|c|}
\hline \multirow{2}{*}{ Tratamiento } & \multicolumn{5}{|c|}{ Altura (m) } & \multirow{2}{*}{$\begin{array}{c}\text { Incremento } \\
2004-2008 \text { (\%) }\end{array}$} \\
\hline & 2004 & 2005 & 2006 & 2007 & 2008 & \\
\hline T1: For. Manejado & 5,3 & 5,7 & 6,1 & 6,6 & 7,0 & 32,1 \\
\hline T2: Silvop. Tradicional & 6,0 & 6,4 & 6,8 & 7,2 & 7,6 & 26,7 \\
\hline T3: Silvop. Fajas & 5,6 & 6,1 & 6,5 & 7,0 & 7,5 & 33,9 \\
\hline
\end{tabular}

Como se observa en el Cuadro $\mathrm{N}^{\circ} 8$ y Figura $\mathrm{N}^{\circ} 4$, las mayores alturas se presentan en tratamiento tradicional y las menores en el forestal, con una situación intermedia para fajas.

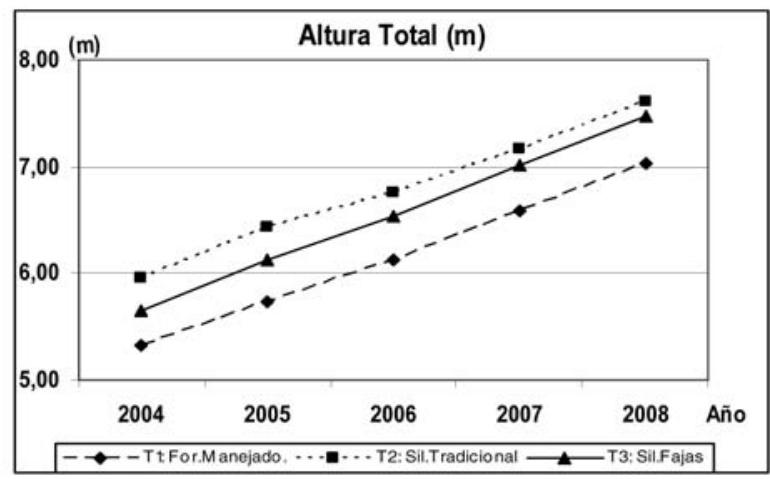

Figura $\mathrm{N}^{\circ} 4$

\section{EVOLUCIÓN DE LA ALTURA MEDIA TOTAL AÑOS 2004 A 2008}

Se probaron tres modelos que ajustan varianzas heterogéneas:

Autoregresivo de orden 1 (AR1)

De Simetría Compuesta (CS)

De Simetría compuesta heterocedástica (CSH).

Los tres modelos alcanzaron los criterios de convergencia requeridos por SAS. EI Test de Levene indicó que hay homogeneidad de varianzas para $\mathrm{H}$ en todos los años evaluados. el mejor modelo corresponde al Modelo CSH, que presenta los menores valores AIC. 


\section{Cuadro $\mathrm{N}^{\circ} 9$ \\ RESULTADOS DEL MODELO CSH PARA H}

\begin{tabular}{|l|c|c|c|c|}
\hline Efecto & GL & DF & Valor $\mathbf{F}$ & Pr $>$ F \\
\hline Tratamiento & 2 & 4 & 8,28 & 0,0378 \\
\hline Tiempo & 4 & 1368 & 280,87 & $<, 0001$ \\
\hline Parcela & 2 & 4 & 0,97 & 0,4548 \\
\hline Tratamiento*Tiempo & 8 & 8 & 0,31 & 0,9612 \\
\hline
\end{tabular}

En el Cuadro $\mathrm{N}^{\circ}$ 9, se observa que el efecto tratamiento y tiempo son significativos para la variable $\mathrm{H}$, y el efecto parcela y tratamiento*tiempo no son significativos.

\section{Cuadro $\mathrm{N}^{\circ} 10$ COMPARACIONES ENTRE TRATAMIENTOS PARA H}

\begin{tabular}{|l|l|c|c|c|c|c|}
\hline Tratamiento & Tratamiento & Estimador & Error Estándar & GL & Valor $\mathbf{t}$ & Pr $>|\mathbf{t}|$ \\
\hline T3: Sil. Fajas & T1: M.Forestal & 0,4161 & 0,1578 & 4 & 2,64 & 0,0578 \\
\hline T3: Sil. Fajas & T2: Sil.Tradicional & $-0,2201$ & 0,1547 & 4 & $-1,42$ & 0,2278 \\
\hline T1: M.Forestal & T2: Sil.Tradicional & $-0,6362$ & 0,1583 & 4 & $-4,02$ & 0,0159 \\
\hline
\end{tabular}

Según los datos expuestos en el Cuadro $N^{\circ} 10$ y la Figura $N^{\circ} 4$, existen diferencias significativas entre los tratamientos $\mathrm{T} 1$ y $\mathrm{T} 2$, y no se presentan diferencias entre T3 con T1 y $\mathrm{T} 2$ para $\mathrm{H}$.

En relación a la altura, que es un buen índice de calidad del sitio (Hush et al., 1982; Smith, 1986), dado que los ensayos han sido instalados en un sitio similar, se esperaría que no hubiera diferencias entre los tratamientos. Según Hush et al. (1982), la densidad afecta levemente la altura de los árboles, y Smith (1986) indica que esta no es afectada por la densidad. En este caso, de acuerdo a los resultados obtenidos al año 2008, no se observa diferencias entre el tratamiento silvopastoral en fajas y los otros dos tratamientos, pero si existen diferencias significativas entre el tratamiento forestal manejado y el silvopastoral tradicional (Cuadro $\mathrm{N}^{\circ}$ 10).

La Figura $N^{\circ} 4$ muestra que en el transcurso de todos los años de evaluación, el tratamiento $\mathrm{T} 2$ es el que ha presentado los mayores valores para $\mathrm{H}$, lo cual puede deberse a pequeñas variaciones de sitio. Otro factor a considerar, es la mayor selección de los árboles


respecto de los tratamientos en fajas y forestal. En el caso del tratamiento en fajas, al mantener las fajas inalteradas para obtener una densidad similar entre los tratamientos silvopastorales, se conservó árboles de menores dimensiones, especialmente al interior de la faja residual. Lo mismo pasa en el tratamiento forestal respecto al tradicional, al conservar más árboles, 816 contra 1514 arb ha ${ }^{-1}$, conservándose árboles de menor dimensión para obtener la densidad requerida. 


\section{-Cobertura de Copa}

La evolución del desarrollo de la cobertura de copa de los tratamientos, de acuerdo a lo expuesto en Cuadro $\mathrm{N}^{\circ} 11$ y Figura $\mathrm{N}^{\circ} 5$, indica un alto cubrimiento de la superficie por la biomasa aérea en el tratamiento forestal, con un $60,24 \%$ bajo la influencia de la copa al año 2008, y de sólo entre $30-32 \%$ en el caso de los tratamientos silvopastorales.

\section{Cuadro $\mathrm{N}^{\circ} 11$}

\section{COBERTURA DE COPA ENTRE LOS AÑOS 2004 A 2008}

\begin{tabular}{|l|c|c|c|c|c|}
\hline \multirow{2}{*}{ Tratamiento } & \multicolumn{4}{|c|}{ Cobertura de Copa (\%) } & Incremento \\
\cline { 2 - 5 } & $\mathbf{2 0 0 4}$ & $\mathbf{2 0 0 6}$ & $\mathbf{2 0 0 7}$ & $\mathbf{2 0 0 8}$ & 2008 (\%) \\
\hline T1: For. Manejado & 26,9 & 36,0 & 55,1 & 60,2 & 123,0 \\
\hline T2: Sil. Tradicional & 14,5 & 21,7 & 31,1 & 32,2 & 121,0 \\
\hline T3: Sil. Fajas & 24,2 & 27,1 & 28,9 & 30,7 & 26,8 \\
\hline
\end{tabular}

La evolución del desarrollo de la copa en los tratamientos T1 y T2 (Figura № 5) muestra que éstos han incrementado la cobertura de copa entre los años 2004 y 2008 sobre un 120\%, contra sólo un $26,8 \%$ en el silvopastoral en fajas. El menor desarrollo de la copa en este último tratamiento, se debe a la mayor competencia de los árboles, al estar concentrados en una menor superficie en la faja. Lo anterior ha impedido la expansión de las ramas hacia el interior de la faja, y solo se ha generado mayor desarrollo de las ramas externas hacia el área de la pradera, sin árboles.

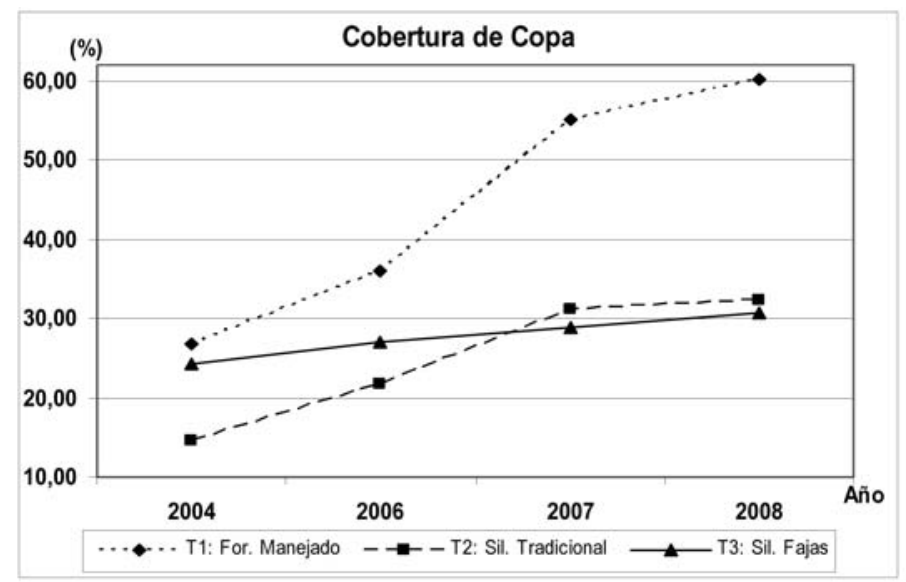

Figura $\mathrm{N}^{\circ} 5$

EVOLUCIÓN DE LA COBERTURA DE COPA POR HECTÁREA, AÑOS 2004 A 2008 
Por otro lado, el diámetro de copa por árbol evolucionó desde 2,05 a 3,07 m en el tratamiento forestal y de 2,28 a 3,41 m en el tratamiento silvopastoral tradicional (Figura $\mathrm{N}^{\circ} 6$ ).

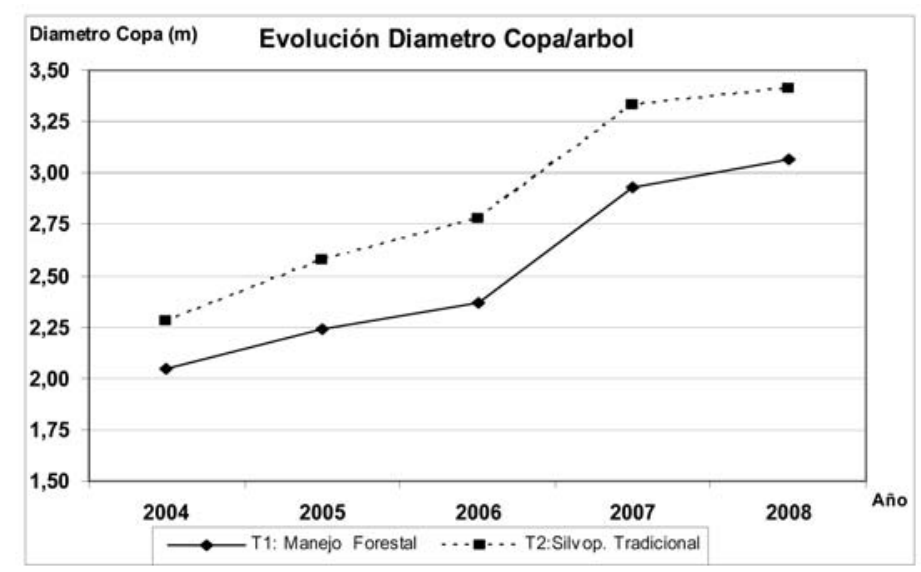

Figura $\mathrm{N}^{\circ} 6$

EVOLUCIÓN DE LA COBERTURA DE COPA POR ÁRBOL AÑOS 2004 A 2008

Para analizar este parámetro, se probaron cuatro modelos que ajustan varianzas heterogéneas:

Autoregresivo de Orden 1 (AR1)

De Simetría Compuesta (CS)

De Simetría Compuesta Heterocedástica (CSH)

No Estructurado (UN)

Los cuatro modelos alcanzaron los criterios de convergencia requeridos por SAS. El Test de Levene indicó que hay homogeneidad de varianzas para Ccopa en todos los años evaluados. El mejor modelo corresponde al modelo UN, que presentó los menores valores AIC.

\section{Cuadro $\mathrm{N}^{\circ} 12$}

\section{RESULTADOS DEL MODELO UN PARA Ccopa}

\begin{tabular}{|l|c|c|c|c|}
\hline Efecto & GL & DF & Valor F & Pr $>$ F \\
\hline Tratamiento & 2 & 4 & 28,48 & 0,0043 \\
\hline Tiempo & 3 & 18 & 454,87 & $<0,0001$ \\
\hline Parcela & 3 & 4 & 0,03 & 0,9739 \\
\hline Tratamiento*Tiempo & 6 & 18 & 83,11 & $<0,0001$ \\
\hline
\end{tabular}


En el Cuadro $N^{\circ} 12$ se observa que el efecto tratamiento, tiempo y tratamiento*tiempo son significativos para la variable Ccopa, y el efecto parcela no es significativo.

Cuadro $N^{\circ} 13$

COMPARACIONES ENTRE TRATAMIENTOS PARA Ccopa

\begin{tabular}{|l|l|c|c|c|c|c|}
\hline Tratamiento & Tratamiento & Estimador & Error Estándar & GL & Valor $\mathbf{~}$ & Pr $>|\mathbf{t}|$ \\
\hline T1: M.Forestal & T3: Sil. Fajas & 16,8542 & 2,8167 & 4 & 5,98 & 0,0039 \\
\hline T1: M.Forestal & T2: Sil.Tradicional & 19,6475 & 2,8167 & 4 & 6,98 & 0,0022 \\
\hline T3: Sil. Fajas & T2: Sil. Tradicional & 2,7933 & 2,8167 & 4 & 0,99 & 0,3775 \\
\hline
\end{tabular}

Los antecedentes expuestos en el Cuadro $\mathrm{N}^{\circ} 13$ y Figura $\mathrm{N}^{\circ} 5$ indican que existen diferencias significativas entre el tratamiento $\mathrm{T} 1$ respecto a los dos tratamientos silvopastorales T2 y T3 para Ccopa, y no se presentan diferencias entre T2 y T3.

Cuadro $\mathrm{N}^{\circ} 14$

\section{COMPARACIONES ENTRE TRATAMIENTO^TIEMPO PARA Ccopa}

\begin{tabular}{|l|l|l|c|}
\hline Tratamiento & Tratamiento & Año & Pr $>|\mathbf{t}|$ \\
\hline T1: M. Forestal & T2: Sil. Tradicional & 2004 & 0,0006 \\
\hline T1: M. Forestal & T3: Sil. Fajas & 2004 & 0,3697 \\
\hline T2: Sil. Tradicional & T3: Sil. Fajas & 2004 & 0,0043 \\
\hline T1: M. Forestal & T2: Sil. Tradicional & 2006 & 0,0001 \\
\hline T1: M. Forestal & T3: Sil. Fajas & 2006 & 0,0077 \\
\hline T2: Sil. Tradicional & T3: Sil. Fajas & 2006 & 0,0864 \\
\hline T1: M. Forestal & T2: Sil. Tradicional & 2007 & $<0,0001$ \\
\hline T1: M. Forestal & T3: Sil. Fajas & 2007 & $<0,0001$ \\
\hline T2: Sil. Tradicional & T3: Sil. Fajas & 2007 & 0,4481 \\
\hline T1: M. Forestal & T2: Sil. Tradicional & 2008 & $<0,0001$ \\
\hline T1: M. Forestal & T3: Sil. Fajas & 2008 & $<0,0001$ \\
\hline T2: Sil. Tradicional & T3: Sil. Fajas & 2008 & 0,6084 \\
\hline
\end{tabular}

La evolución de la cobertura de copa en el tiempo (Cuadro $N^{\circ} 14$ ) indica que desde el inicio existieron diferencias significativas entre el tratamiento forestal y el silvopastoral tradicional, y sin diferencias en el primer año con el tratamiento en fajas. A partir del segundo año, las diferencias se manifestaron en relación a los dos tratamientos silvopastorales en relación al tratamiento forestal, lo cual se ha mantenido hasta el último año de medición. No han existido diferencias entre los tratamientos silvopastorales, salvo el primer año. El tratamiento silvopastoral tradicional, producto de un agresivo raleo el año 2003, registró una cobertura de copa bastante menor al de fajas (14,5 contra $24,1 \%)$, situación que se revirtió rápidamente 
(Figura $\mathrm{N}^{\circ}$ 5) dado el mayor espacio que tenían los árboles en T2 para expandir sus copas. Luego, al llegar a la tercera y cuarta temporada, estos valores se estabilizaron en alrededor de $32 \%$ de Ccopa, valor similar a tratamiento T3.

\section{-Cociente de Forma Local}

El cociente de forma local fue calculado en base a las mediciones de DAP y $D_{3,2 m}$, realizadas durante el verano 2008-2009. Los resultados expuestos en Cuadro № 15 indican que los mayores valores se presentan en T2, con 0,758.

\section{Cuadro $\mathrm{N}^{\circ} 15$ \\ COCIENTE DE FORMA LOCAL AÑO 2009}

\begin{tabular}{|c|c|}
\hline Tratamiento & CFL \\
\hline T1. For.Manejado & 0,726 \\
\hline T2. Silv.Tradicional & 0,758 \\
\hline T3. Silv.Fajas & 0,729 \\
\hline
\end{tabular}

Para realizar el análisis estadístico, se usó un análisis de varianza tradicional, dado que se cumplen los criterios básicos de este tipo de análisis estadístico.

De acuerdo a lo expuesto en Cuadro $N^{\circ} 16$, no existen diferencias significativas para cociente de forma local entre los tratamientos silvopastorales y el tratamiento forestal manejado para la temporada analizada.

\section{Cuadro $\mathrm{N}^{\circ} 16$ \\ ANÁLISIS DE VARIANZA PARA COCIENTE DE FORMA LOCAL}

\begin{tabular}{|l|c|c|c|}
\hline Tratamiento & Medias & $\mathbf{n}$ & $\mathbf{p}<\mathbf{0}, \mathbf{0 5}$ * \\
\hline T1. For. Manejado & 0,726 & 3 & $\mathrm{a}$ \\
\hline T3. Sil.Fajas & 0,729 & 3 & $\mathrm{a}$ \\
\hline T2. Sil.Tradicional & 0,758 & 3 & $\mathrm{a}$ \\
\hline
\end{tabular}

*: Letras distintas indican diferencias significativas.

Dado que el diámetro de los árboles está influenciado por la densidad, aunque no existen diferencias significativas, el mayor CFL atribuible al tratamiento silvopastoral tradicional indica que estos árboles son levemente más cilíndricos que en los otros dos tratamientos. Se deberá esperar su evolución en el futuro, para así analizar el efecto de la densidad sobre éste cociente en el tiempo.

\section{-Diámetro de Ramas}

El diámetro de ramas fue medido sólo en las dos últimas temporadas de evaluación. Los resultados indican que los mayores valores se presentan en T1 y T3, con 5,1 y 5,0 cm, y 4,5 $\mathrm{cm}$ en T2 (Cuadro $\mathrm{N}^{\circ} 17$ ). 
Cuadro $\mathrm{N}^{\circ} 17$

DIÁMETRO DE RAMAS AÑOS 2007 Y 2008

\begin{tabular}{|l|c|c|c|}
\hline \multirow{2}{*}{ Tratamiento } & \multicolumn{2}{|c|}{$\begin{array}{c}\text { Diámetro de Ramas } \\
\text { (cm) }\end{array}$} & \multirow{2}{*}{$\begin{array}{c}\text { Incremento } \\
\text { (\%) }\end{array}$} \\
\cline { 2 - 3 } & $\mathbf{2 0 0 7}$ & $\mathbf{2 0 0 8}$ & 3,16 \\
\hline T1: For. Manejado & 4,9 & 5,1 & 2,89 \\
\hline T2: Sil. Tradicional & 4,3 & 4,5 & 5,63 \\
\hline T3: Sil. Fajas & 4,8 & 5,0 & \\
\hline
\end{tabular}

Dado que el diámetro de ramas está influenciado por la densidad y la luz que llega a la copa, se habría esperado que el tratamiento forestal fuera el de menor diámetro, lo cual se obtuvo a la inversa (Figura $\mathrm{N}^{\circ} 7$ ).

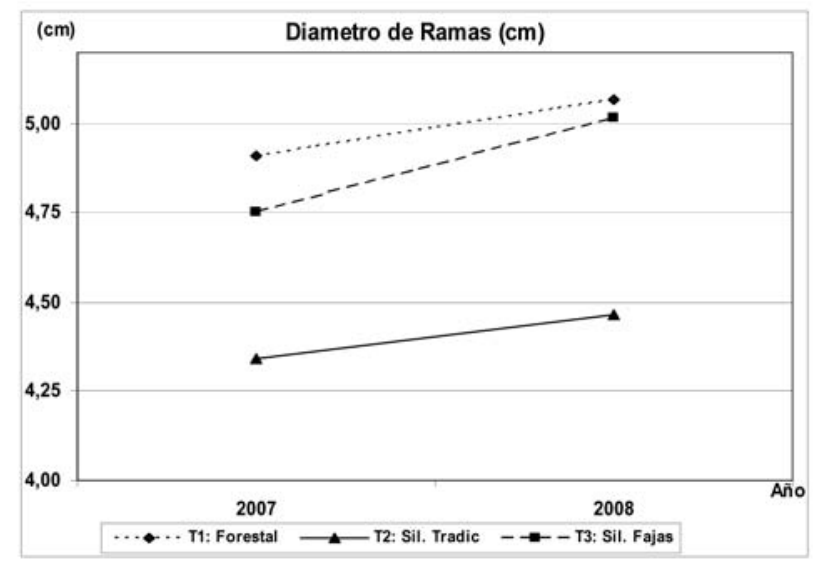

Figura $N^{\circ} 7$

EVOLUCIÓN DIÁMETRO DE RAMAS AÑOS 2007 A 2008

Dado que no se realizaron las mediciones iniciales en el año 2004 a 2006, solo se evaluaron las dos últimas temporadas.

Para analizar este parámetro se probaron tres modelos que ajustan varianzas heterogéneas:

Autoregresivo de Orden 1 (AR1)

De Simetría Compuesta (CS)

De Simetría Compuesta Heterocedástica (CSH).

Los tres Modelos alcanzaron los criterios de convergencia requeridos por SAS. El Test de Levene indicó que hay homogeneidad de varianzas para DR en todos los años evaluados.

El mejor modelo corresponde al Modelo CS, que presenta los menores valores AIC. 


\section{Cuadro $\mathrm{N}^{\circ} 18$ \\ RESULTADOS DEL MODELO CS PARA DR}

\begin{tabular}{|l|c|c|c|c|}
\hline Efecto & GL & DF & Valor F & Pr $>$ F \\
\hline Tratamiento & 2 & 4 & 0,53 & 0,6263 \\
\hline Tiempo & 1 & 116 & 1,40 & 0,2384 \\
\hline Parcela & 2 & 4 & 0,11 & 0,8980 \\
\hline Tratamiento*Tiempo & 2 & 116 & 0,23 & 0,7938 \\
\hline
\end{tabular}

En el Cuadro № 19 se observa la ausencia de diferencias estadísticas significativas para el efecto tratamiento, tiempo, parcela y tratamiento*tiempo para DR.

\section{Cuadro $\mathrm{N}^{\circ} 19$}

\section{COMPARACIONES ENTRE TRATAMIENTOS PARA DR}

\begin{tabular}{|l|l|c|c|c|c|c|}
\hline Tratamiento & Tratamiento & Estimador & Error Estándar & GL & Valor $\mathbf{t}$ & $\operatorname{Pr}>|\mathbf{t}|$ \\
\hline T3: Sil. Fajas & T1: M.Forestal & $-0,3099$ & 0,6659 & 4 & $-0,47$ & 0,6658 \\
\hline T3: Sil. Fajas & T2: Sil.Tradicional & 0,3765 & 0,6460 & 4 & 0,58 & 0,5913 \\
\hline T1: M.Forestal & T2: Sil.Tradicional & 0,6864 & 0,6713 & 4 & 1,02 & 0,3643 \\
\hline
\end{tabular}

Como se expone en los Cuadros $\mathrm{N}^{\circ} 18$ y $\mathrm{N}^{\circ} 19$, y en la Figura $\mathrm{N}^{\circ} 7$, no existen diferencias estadísticas significativas entre los tres tratamientos. No obstante, se observa un menor diámetro de ramas para el tratamiento tradicional frente a los otros dos tratamientos.

\section{-Cobertura de Desechos}

En los tratamientos con presencia de árboles, se generaron desechos producto de las faenas de poda y raleo realizada el año 2004, los cuales fueron ordenados con diferentes estrategias de acuerdo al tratamiento (Cuadro $N^{\circ} 20$ ). Entre los tratamientos silvopastorales, el tratamiento en fajas contiene una mayor proporción de área cubierta con desechos en relación al tradicional. Lo anterior, dado que el ordenamiento de desechos en el tratamiento en fajas, posterior al manejo del año 2004, se ubicó en la superficie de la faja cubierta con árboles, dejando limpias las áreas de praderas. 


\section{Cuadro $\mathrm{N}^{\circ} 20$ \\ COBERTURA DE DESECHOS FORESTALES EN LOS TRATAMIENTOS}

\begin{tabular}{|l|c|c|c|}
\hline Tratamiento & $\begin{array}{c}\text { Cobertura de Desechos } \\
\text { Forestales (\%) }\end{array}$ & $\begin{array}{c}\text { Tipo de } \\
\text { Ordenamiento }\end{array}$ & $\begin{array}{c}\text { Ancho Faja de } \\
\text { Desechos (m) }\end{array}$ \\
\hline T1. For. Manejado & 80,00 & $\begin{array}{c}\text { Desechos esparcidos } \\
\text { uniformemente en el terreno }\end{array}$ & - \\
\hline T2. Sil. Tradicional & 25,87 & $\begin{array}{c}\text { Desechos ordenados en fajas } \\
\text { cada 20 metros }\end{array}$ & 5,17 \\
\hline T3. Sil. Fajas & 31,03 & $\begin{array}{c}\text { Desechos ordenados bajo la faja } \\
\text { de árboles cada 21 m. }\end{array}$ & 6,86 \\
\hline
\end{tabular}

Las fajas de árboles con desechos quedaron a una distancia una de otra de $21 \mathrm{~m}$; en el caso de tratamiento tradicional, las fajas de desechos fueron ordenadas cada $20 \mathrm{~m}$. En el tratamiento forestal, éstos fueron distribuidos homogéneamente.

\section{-Daños en los Árboles}

Pastoreo

En general, durante las cuatro temporadas de evaluación, no se produjeron daños importantes producto del tránsito o ramoneo por animales, dado que los árboles al comienzo de la investigación tenían una altura superior a los $5,5 \mathrm{~m}$, y con corteza madura. La poda de $2 \mathrm{~m}$ no permitió el ramoneo de las ramas y acículas. Solo se presentaron algunos daños menores en el tratamiento en fajas, menor al 0,5\%, en áreas de tránsito entre fajas de árboles por donde los animales se movían de un área de pradera hacia la otra, produciéndose desprendimiento de corteza por fricción producidos por el cuerpo del animal o daño por cuernos.

Viento

No se produjeron daños importantes por efecto del viento, después de abrir el bosque por raleo desde 1.514 a $800 \mathrm{arb}^{*}$ ha $^{-1}$ en el tratamiento forestal, y a 357-400 arb * ha-1 en los tratamientos silvopastorales. En el tratamiento silvopastoral tradicional se produjo un daño por inclinación de árboles del 2,0\% producto del viento, mientras que en los tratamientos en fajas y forestal no fueron observados daños.

\section{DISCUSIÓN}

El efecto de un manejo de los árboles con fines silvopastorales, con densidades bajas, tiene un importante efecto sobre el desarrollo de las variables forestales. Por un lado, al disminuir la densidad inicial desde 1.514 a 350-400 árboles, aproximadamente en los diseños silvopastorales, se reduce fuertemente el $A B$ (Figura $N^{\circ} 3$ ), pero se aumenta el incremento en diámetro de los árboles en dichos tratamientos (Figura $N^{\circ} 2$ ), lo cual coincide con lo expuesto por Shepherd y Forrest (1973), Laar (1982), y Sotomayor (1990a). Es decir, se tienen menos árboles, pero con un mayor diámetro, lo cual originará en el futuro árboles de mayores 
dimensiones, obteniéndose una mayor productividad en madera aserrada por árbol, y trozos de mayores dimensiones, podados y sin poda, para ser destinados a producción de madera aserrada y tableros. No obstante, se obtendrá una menor producción de volumen total por hectárea (Bennet 1971; Jones 1977; Sotomayor 1980; Sotomayor y Cabrera 2008).

Otro aspecto a considerar, para el manejo futuro de los tratamientos silvopastorales y forestal, es la disminución del ICA del DAP a partir del año 2007 (Figura № 8), lo cual indica la necesidad de reducir la densidad con raleo, para estimular su crecimiento.

Por otro lado, los tratamientos con una mayor densidad, como es el caso de tratamiento forestal, generan un mayor volumen de madera sólida, razón por lo que estos tratamientos están diseñados para producción de biomasa maderera, con trozos de menores dimensiones. El sistema forestal manejado, obtendrá un interesante volumen de madera libre de defectos de las primeras trozas podadas, y una mayor proporción de madera con usos industriales de baja calidad, en relación a tratamientos silvopastorales, como madera destinada a pulpa, leña o postes, lo cual está de acuerdo a lo expuesto por Bennet (1971), Shepherd y Forrest (1973), y Sotomayor y Cabrera (2008).

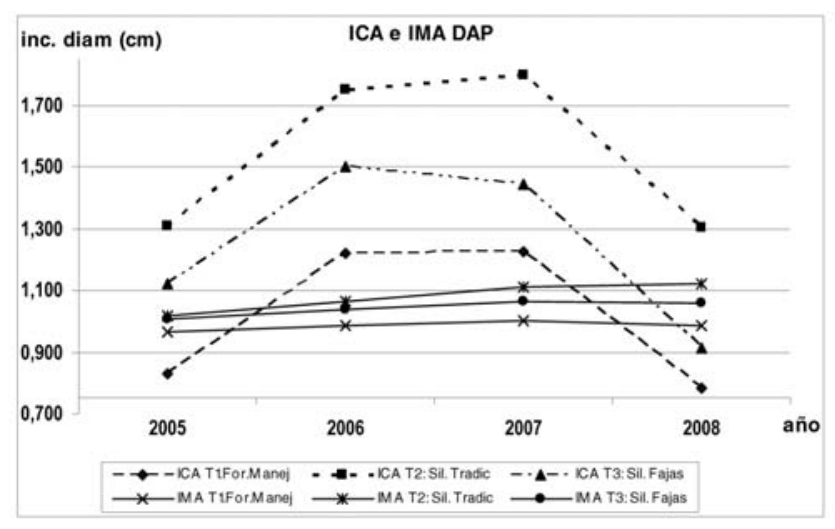

\section{Figura $\mathrm{N}^{\circ} 8$ \\ ICA E IMA DEL DAP POR TRATAMIENTO}

En el futuro, se espera que las diferencias en $A B$ entre los tratamientos estudiados disminuyan, producto del esquema de manejo planificado (con raleos y podas) expuesto en metodología general, y del incremento en diámetro en los tratamientos silvopastorales. Esto originará que las diferencias en volumen total al final de la rotación también disminuirán, con un aumento de los productos podados y de mayor valor en los tratamientos silvopastorales. Lo anterior, originará a su vez que el valor de la madera por árbol proporcionalmente será mayor en los tratamientos silvopastorales que en el tratamiento forestal, lo cual coincide con lo expuesto por Sotomayor y Cabrera (2008).

Un factor que es importante de considerar en un manejo silvopastoral, es el diámetro de ramas. A menor densidad inicial, o producto de raleos tempranos y agresivos, se produce un 
fuerte incremento del diámetro de ramas en los sectores no podados (Bennet 1971; Sotomayor 1980), y como consecuencia un aumento del centro nudoso (Bennet 1971; Batíni et al. 1983), lo cual puede desclasificar estas trozas para uso de madera aserrada de calidad. Por ello es importante, en tratamientos silvopastorales, el podar lo más tempranamente posible y a alturas apropiadas para obtener trozos libre de nudos, y así reducir la cantidad de desechos. Aunque en este estudio no se obtuvieron diferencias significativas en esta variable, por el corto periodo de evaluación, otros estudios si lo han indicado. Se ha observado que el trozo siguiente al sector podado, presenta un fuerte incremento en el diámetro de ramas (Sotomayor 1980, 1990a), lo cual produce "coronas" de ramas de gran tamaño, lo que imposibilita su uso para madera aserrada por una alta presencia de nudos en la madera.

Con respecto a la evolución en el desarrollo de la copa, en el presente trabajo se observó un fuerte incremento en la cobertura de copa por unidad de superficie a medida que la densidad aumenta, lo cual coincide con lo expresado por Lewis et al. (1985). El mayor incremento en cobertura de copa se presentó en el tratamiento forestal manejado, tratamiento que cuenta con mayor número de árboles, y luego en el silvopastoral tradicional. En ambos tratamientos, hasta la fecha de evaluación el año 2008, los árboles han tenido suficiente espacio para el desarrollo de las copas. En el caso del tratamiento en fajas, se observa un desarrollo menor de su copa, comparada con el tratamiento tradicional, dada la fuerte competencia de los árboles en el área de crecimiento (faja), donde existe un distanciamiento medio entre árboles de 2,2 m contra 5,3 $\mathrm{m}$ en el silvopastoral tradicional.

\section{CONCLUSIONES}

Los árboles de Pinus contorta, manejados bajo ordenamiento silvopastoral, se comportaron adecuadamente bajo densidades bajas, entre 350-400 arb ha-1 y raleos fuertes, en relación al efecto del viento, no presentándose daños significativos por este factor.

A su vez, los árboles manejados bajo esquemas silvopastorales, presentaron:

- Un buen desarrollo en DAP, en relación al tratamiento forestal, con diferencias significativas en el tiempo, y con un mayor DAP en el tratamiento silvopastoral tradicional, y el menor DAP en el tratamiento forestal.

- Una menor área basal, producto de una menor densidad, en relación a tratamiento forestal.

- Una menor cobertura de copa por unidad de superficie, pero un mayor diámetro de copa por árbol.

- Similar cociente de forma local, en relación al tratamiento forestal.

- Diámetro de ramas sin diferencias significativas, entre tratamientos.

No se presentaron daños significativos en los árboles de Pinus contorta, bajo ordenamiento silvopastoral, por efecto del manejo animal. Tampoco se presentaron daños por efecto del viento, después de la apertura del dosel con raleos intensos el año 2003. 


\section{REFERENCIAS}

Anderson, G., Moore, R. and Jenkins, P., 1988. The integration of pasture, livestock and widely-spaced pine in South Western Australia. Agrofor. Syst. 6:195-211.

Arnold, J. E. M., 1983. Economic considerations in agroforestry projects. Agrofor.Syst. 1:229311.

Batíni, F. E., Anderson, G. W. and Moore, R., 1983. The practice of agroforestry in Australia. pp. 233-246. En: Foothills for Food and Forest. Ed. By Hannaway D.B. Timper Press and Oregon State Univ. Beaverton, Oregon.

Bennett, F. A., 1971. The role of thinning and some other problems in Management of Slash Pine plantations. USDA Forest Service, Res. Paper SE-86.

Carrero O., Jerez M. Macchiavelli R., Orlandoni G. y Stock J., 2008. Ajuste de curvas de indice de sitio mediante modelos mixtos para plantaciones de Eucalyptus urophylla en Venezuela. Interciencia v.33 (4).

Doesher, P. S., Tesh, S. D. and Alejandro-Castro, M., 1987. Livestock grazing: A silvicultural tool for plantation establishment. J. For. 83: 29-37.

Garret, H. E. and Buck, L., 1997. Agroforestry practice and policy in the United States of America. Forest Ecol. and Manag. 91:5-15.

Garret, H. E., Kerley, M. S., Ladyman, K. P., Walter, W. D., Godsey, L. D., Van Sambeek. J. W. and Brauer, D. K., 2004. Hardwood silvopasture management in North America. Agrofor. Syst. 61:21-33.

Hush B., Millar C.I. and Beers T.W., 1982. Forest Mensuration. Pub. John Wiley \& Sons. New York, United States of America.

INE, 1997. VI Censo Nacional Agropecuario. Santiago, Chile.

INFOR, 2008. Boletín Estadístico 121: Anuario Forestal 2008. Instituto Forestal, Santiago, Chile.

Jones Jr., E. P., 1977. Precommercial thinning of naturally seeded slash pine increases volume and monetary returns. USDA Forest Service, Res.Paper SE-164.

Krueger W. C., 1987. Pacific Northwest forest plantations and livestock grazing. J. For. 83:3031.

Laar, A. V., 1982. The response of Pinus radiata to initial spacing. South Afric.For.J. 121:5262

Lewis C.E., 1973. Integrating management of forest and range resources. pp 69-70. In: Range 
resources of the Southeastern United States. The Amer. Soc. Agro., Pub № 21. Madison, Wisconsin.

Lewis, C., Tanner, G. and Terry, W., 1985. Double vs. Single row pine plantation for wood and forage production. South. J. App. For. 9:55-61.

Longhurst, W. M., 1983. Wildlife interactions with domestic animals and forests. In: Foothills for Food anf Forest. Ed. by Hannaway D.B. Oregon State Univ. Timber Press, Beaverton, Oregon. Pp. 309-320.

Mead, D., 2009. Biophysical interactions in silvopastoral systems: A New Zealand perspective. En: Actas del $1^{\text {er }}$ Congreso Nacional de Sistemas Silvopastoriles. Posadas, Misiones Argentina. Ed. Instituto Nacional de Tecnología Agropecuaria, Buenos Aires, Argentina. pp. 3-9.

Monfore, J. D., 1983. Livestock, a useful tool for vegetation control on Ponderosa Pine and Lodgepole Pine plantations. Pp.105-107. In: Forestland Grazing. Ed: by Roche, Jr. B.F. and Baumgrtner D.M., and Cooperative Extension, Wash. State Univ. Spokane, Washington.

Murgeitio, E., 2009. Aspectos relacionados con la sustentabilidad social y ambiental de los sistemas silvopastoriles en América tropical. En: Actas del $1^{\mathrm{er}}$ Congreso Nacional de Sistemas Silvopastoriles. Posadas, Misiones Argentina. Ed. Instituto Nacional de Tecnología Agropecuaria, Buenos Aires, Argentina. Pp. 66-69.

Shepherd, K. R. and Forrest, W. G., 1973. Growth of Radiata Pine following thinning. Commonw. For Rev. 52:133-141.

Smith, D. M.,1986. The Practice of Silviculture. Eight Edition. John Wiley \& Sons. New York.

Sotomayor. A., 1980. Espaciamiento en plantaciones de Pinus radiata D.Don. Tesis presentada como parte de los requisitos para optar al título de Ingeniero Forestal, Facultad de Ciencias Forestales, Universidad de Chile, Santiago, Chile, 1980.

Sotomayor, A., 1989. Sistemas silvopastorales y su manejo. Documento técnico № 42 . Revista Chile Forestal, Diciembre 1989. CONAF. 8p.

Sotomayor, A., 1990a. Sistemas silvopastorales y su manejo. Chile Agrícola 157:203-206.

Sotomayor, A., 1990c. Bosques y forrajeras pueden complementarse (III Parte). Chile Agrícola 159:273-277.

Sotomayor, A. y Cabrera, C., 2008. Análisis de un sistema silvopastoral con Pinus radiata D. Don, asociado con ganado ovino en la zona mediterránea costera central de Chile. Ciencia e Investigación Forestal, Instituto Forestal. Volumen 14 № 2. pp: 269-286.

Sotomayor, A., 2009. Sistemas silvopastorales, alternativa productiva para un desarrollo sustentable de la agricultura en Chile. En: Actas del $1^{\text {er }}$ Congreso Nacional de Sistemas Silvopastoriles. Posadas, Misiones Argentina. Ed. Instituto Nacional de Tecnología Agropecuaria, 
Buenos Aires, Argentina. pp.26-47.

Teuber, O., y Ganderatz, S., 2009. Características geográficas y edafoclimáticas de la región de Aysén. En: Sistemas Agroforestales para la región de Aysén: Cortinas Cortaviento y Silvopastoreo. pp: 85-128. Ed: Teuber O. Instituto de Investigaciones Agropecuarias e Instituto Forestal. Coyhaique, Chile.

Tustin, J. R., Knowles, R. L. and Klomp, B. K., 1979. Forest farming: A multiple land-use production system in New Zealand. For. Ecol. \& Mgt. 2:169-189. 